\title{
Plasma rich in growth factors and bone formation: a radiological and histomorphometric study in New Zealand rabbits
}

\section{Francisco Molina-Miñano(a) \\ Pía López-Jornet ${ }^{(b)}$ \\ Fabio Camacho-Alonso ${ }^{(c)}$ \\ Vicente Vicente-Ortega ${ }^{(d)}$}

(a) $D D S$; (b)PhD, MD, DDS; (c) PhD, DDS

- Department of Oral Medicine, School of Medicine and Dentistry, University of Murcia, Spain.

(d) PhD, MD, Service of Pathology, School of Medicine and Dentistry, University of Murcia, Spain.
Corresponding author:

Pía López-Jornet

Clínica Odontológica Universitaria

Hospital Morales Meseguer

Adv. Marques de los velez, s/n

Murcia 30008 - Spain

E-mail:majornet@um.es

\begin{abstract}
A radiographic and histomorphometric study was conducted on the influence of autologous plasma rich in growth factors (PRGF) upon bone healing in surgically created defects in rabbits. Radiographically, bone regeneration was significantly greater with the use of PRGF after one month $(\mathrm{p}=0.005)$, though no differences were recorded after the second month. In the histomorphometric analysis one month after surgery, the defects filled with autologous bone plus PRGF showed a greater percentage of neoformed bone $(35.01 \pm 5.31)$ than the control defects $(22.90 \pm 12.23)$, though the differences were not significant. Two months after surgery, the defects filled with autologous bone showed greater regeneration $(46.04 \pm 10.36 \%)$ than the control defects $(30.59 \pm 5.69 \%)$, though the differences were not significant. The application of PRGF in the bone defects produced in New Zealand rabbits exerted a limited effect on local bone formation.
\end{abstract}

Descriptors: Bone and bones; Intercellular signaling peptides and proteins; Rabbits; Radiography; Histology. 


\section{Introduction}

Platelet-rich preparations constitute a relatively new biotechnology for the stimulation and acceleration of tissue healing and bone regeneration. ${ }^{1-3}$ Platelet-rich plasma (PRP) is defined as a concentration of autologous platelets in a small volume of plasma, and is considered to be a rich source of autologous growth factors. It was first introduced by Marx et al. ${ }^{3}$ (1998) in combination with autologous bone grafts for the reconstruction of mandibular defects. The contribution of PRP formulations to the bone healing process is thought to be based on the growth factors (GFs) contained in such formulations. In 1999, Anitua ${ }^{4}$ proposed the use of plasma rich in growth factors (PRGF), where the platelets contain growth factors such as TGF-B1, VEGF, and IGF. These proteins intervene in functions such as directed cell migration (chemotaxis) and in cellular differentiation and proliferation, all of which are key events in repair and regeneration processes.

The biological effects of the different GFs cannot yet be defined in terms of a specific concentration. As a result, it is not known how much of a given growth factor is needed to accelerate the bone healing process. ${ }^{5,6}$

Marx et al. ${ }^{3}$ (1998) used PRP together with autologous bone grafts in mandibular reconstructions, and showed the radiographic density to be greater in grafts to which PRP had been added than in grafts without added PRP, after 6 months. The same study found the radiographic maturation rate to be 1.622.16 times greater in the PRP-added grafts than in the grafts without added PRP. Afterwards, a number of studies ${ }^{7-9}$ have been published on the use of PRP to enhance many different combinations of autologous and/or exogenous graft materials.

Aghaloo et al..$^{10}$ (2002) performed bicortical defects in rabbit skulls, and evaluated the effects of PRP upon regeneration of the lesions. No significant radiographic or morphometric differences were found in relation to bone formation. In another experimental model in dogs, Gerard et al. ${ }^{11}$ (2006) reported that PRP appeared to enhance early autologous graft healing. However, after two months this effect was no longer significant, and PRP did not change the rate at which new bone was formed. Furthermore, no increase in trabecular density was observed in the PRP-added grafts. The controversy found in the literature regarding the use of this technique is probably related to a lack of standardization in the different PRP formulations, and in the protocols, experimental models and surgical techniques employed. ${ }^{11-13}$

The present study comprises a radiographic and histomorphometric analysis of the influence of PRGF and autologous bone grafting upon bone healing in surgically created defects in New Zealand rabbits, after one and two months.

\section{Material and Methods}

The study involved 20 adult male albino New Zealand rabbits, with a mean weight of $3,662 \mathrm{~g}$, and was carried out in the laboratory of experimental surgery of the University of Murcia (Spain), following approval from the local ethics board. The experiments were conducted in accordance with the guidelines laid down by the European Communities Council Directive of November, 241986 (86/609/EEC). The animals were anesthetized with a mixture of $60 \%$ ketamine and $40 \%$ xylazine. Following disinfection of the zone and under aseptic conditions, we performed an incision in the anterointernal proximal zone of the tibia, since this access is scantly traumatic and causes limited bleeding. The muscle layers were dissected until the periosteum was reached. Four 4-mm diameter defects were created with a trephine bur under copious irrigation. Two bone defects were made in each hind tibia: in the right tibia, one defect was used as control, while PRGF was applied to the other. In the left tibia, the previously crushed autologous bone obtained from the right tibia was used to fill both osteotomy defects, with the addition of PRGF in one of the defects. The wounds were closed with resorbable sutures (Laboratorios Lorca Marín, S.A. Murcia, Spain).

In order to obtain plasma rich in growth factors, a minimum of $10 \mathrm{ml}$ of blood are required. The blood was obtained via cardiac aspiration. Immediately after collection, the blood was placed in sterile 5 -ml extraction tubes, with $3.8 \%$ sodium citrate as anticoagulant. The tubes were placed in a centrifuge (BTI PRGF ${ }^{\circledast}$ System III; Biotechnology Institute, S.L., Álava, Spain) and centrifuged at 1,800 rpm 
for 8 minutes, thereby separating the different blood phases. The plasma poor in growth factors (PPGF) located in the upper part of the plasma in the tube was eliminated with $500 \mu \mathrm{l}$ pipettes. PPGF has the least amount of platelets in the plasma. The PRGF $(0.5 \mathrm{ml})$ was located on top of the white cells series. The PRGF was separated with $500 \mu$ pipettes and transported to an independent tube. Next, the PRGF was activated using $50 \mu \mathrm{l}$ of $10 \%$ calcium chloride.

The mixture of PRGF and calcium chloride was left at room temperature for 10 minutes, until a consistent and easy to handle gelatinous layer was formed. This layer was close to the white cells.

The gelatinous layer was then mixed with autologous bone and placed in the bone defects.

The 20 animals were randomized to two groups: Group $1(\mathrm{n}=10)$ (sacrificed by $\mathrm{CO}_{2}$ inhalation after one month) and Group $2(\mathrm{n}=10)$ (sacrificed after two months). All animals were subjected to the procedure described above, which was followed by radiographic, histological and morphometric analyses.

The radiographic evaluation was carried out with an intraoral X-ray apparatus $(60 \mathrm{kVp}, 7 \mathrm{~mA}$, $0.05 \mathrm{~s})$, generating and processing the images with a radiovisiographic system (Trophy ${ }^{\circledR}$, Eastman Kodak Co., Rochester, NY) and personal computer using Trophy Windows v.5.06 software. The osteotomy defects were classified and scored according to Pryor $^{14}$ as follows:

- Grade 1 - No/limited bone filling: $<25 \%$ of the defect width was filled with bone;

- grade 2 - Partial bone filling: $>25 \%$ and $\leq 90 \%$ of the defect width was filled with bone;

- grade 3 - Complete bone filling: $>90 \%$ of the defect width was filled with bone.

For the light microscopy analysis, the tissues were decalcified in $4.13 \%$ EDTA for 30 days, cut in half and processed for embedding in paraffin. The $5 \mu \mathrm{m}$-thick sections were then stained with hematoxylin-eosin and the Masson trichromic stain.

To perform the histomorphometric analysis, light micrographs ( $\mathrm{x} 6$ magnification) of the biopsy sections were obtained with a digital camera and analyzed using MIP- $4{ }^{\circledR}$ histomorphometry software (Digital Image, Barcelona, Spain). The amount of new bone formed was calculated as a percentage of the total area of the original defect.

Evaluation of the radiographic images and histomorphometric results was carried out by an investigator blinded to the treatment involved in each case.

The data were analyzed using the SPSS v.12.0 statistical package (SPSS ${ }^{\circledR}$ Inc., Chicago, IL, USA). A descriptive study was made of each variable. The Kolmogorov-Smirnov normality test and Levene variance homogeneity test were applied, and if the data showed a skewed distribution, analysis was carried out using a nonparametric ranking test. Statistical significance was accepted for $\mathrm{p} \leq 0.05$.

\section{Results}

Of the 20 rabbits included in the study, 2 were lost to the effects of analysis because of fracture of the operated limb (due to unknown causes). In addition, 6 specimens were damaged in the course of sample manipulation and sectioning, and 6 were damaged during histological processing. A final total of 50 samples could be evaluated.

Evaluation of the standardized digital radiographic images showed that one month after surgery, most of the defects in which PRGF was applied were either partially $(50 \%)$ or completely filled $(37.5 \%)$, while in the control group most of the defects remained partially vacant $(62.5 \%)$. The differences were statistically significant. After two months, the PRGF treated defects showed a greater percentage of radiographically manifest bone regeneration $(66.67 \%)$, though without statistically significant differences (Table 1).

In the histomorphometric analysis one month after surgery, the defects filled with autologous bone plus PRGF showed a greater percentage of neoformed bone $(35.01 \pm 5.31)$ than the control defects $(22.90 \pm 12.23)$, though the differences were not significant. Two months after surgery, the defects filled with autologous bone showed greater regeneration $(46.04 \pm 10.36 \%)$ than the control defects $(30.59 \pm 5.69 \%)$, though once again the differences were not significant (Table 2, Figures 1 and 2).

\section{Discussion}

PRP has been associated with grafts for the purpose of improving bone healing. In the present 
Table 1 - Radiographic analysis of bone regeneration. Pearson's chi-squared test.

\begin{tabular}{|c|c|c|c|c|c|c|}
\hline \multirow{2}{*}{$\begin{array}{l}\text { Observation } \\
\text { period month }\end{array}$} & \multirow[t]{2}{*}{ Group } & \multirow[t]{2}{*}{ Total } & \multicolumn{3}{|c|}{$\begin{array}{l}\text { Radiographic bone regeneration } \\
\qquad \mathrm{n}(\%)\end{array}$} & \multirow[t]{2}{*}{ p-value } \\
\hline & & & Grade 1 & Grade 2 & Grade 3 & \\
\hline \multirow{4}{*}{1} & PRGF & 8 & $1(12.50)$ & $4(50)$ & $3(37.50)$ & \multirow{4}{*}{0.005} \\
\hline & Control & 8 & $5(62.50)$ & $3(37.50)$ & $0(0)$ & \\
\hline & Autologous bone + PRGF & 5 & $0(0)$ & $3(60)$ & $2(40)$ & \\
\hline & Autologous bone & 5 & $3(60)$ & $2(40)$ & $0(0)$ & \\
\hline \multirow{4}{*}{2} & PRGF & 6 & $0(0)$ & $2(33.33)$ & $4(66.67)$ & \multirow{4}{*}{0.006} \\
\hline & Control & 6 & $2(33.33)$ & $4(66.67)$ & $0(0)$ & \\
\hline & Autologous bone + PRGF & 6 & $0(0)$ & $3(50)$ & $3(50)$ & \\
\hline & Autologous bone & 6 & 2 (33.33) & $4(66.67)$ & $0(0)$ & \\
\hline
\end{tabular}

Radiographic evaluation scored ${ }^{14}$ : Grade 1 - No/limited bone filling: < 25\% of the defect width was filled with bone; Grade 2 - Partial bone filling: $>25 \%$ and $\leq 90 \%$ of the defect width was filled with bone; Grade 3 - Complete bone filling: $>90 \%$ of the defect width was filled with bone.

Note: 14 samples from the one month observation period, 2 from the PRGF group, 2 from the control group, 5 from the Autologous bone + PRGF group, and 5 from the autologous bone group were lost during sample preparation. 16 samples from the two month observation period, 4 from the PRGF group, 4 from the control group, 4 from the Autologous bone + PRGF group, and 4 from the autologous bone group were lost during sample preparation.

Table 2 - Histomorphometry: new bone formed as a $\%$ of the total area of the original surgical defect. Kruskal-Wallis test.

\begin{tabular}{c|l|c|c}
\hline $\begin{array}{c}\text { Observation } \\
\text { period month }\end{array}$ & \multicolumn{1}{|c|}{ Group } & $\begin{array}{c}\text { Histomorphometry } \\
\text { mean } \pm \text { sd }\end{array}$ & p-value \\
\hline \multirow{2}{*}{1} & PRGF & $28.58 \pm 11.22$ & \\
\cline { 2 - 3 } & Control & $22.90 \pm 12.23$ & \multirow{2}{*}{0.658} \\
\cline { 2 - 3 } & Autologous bone + PRGF & $35.01 \pm 5.31$ & \\
\cline { 2 - 3 } & Autologous bone & $34.61 \pm 16.93$ & \\
\hline \multirow{2}{*}{2} & PRGF & $41.02 \pm 9.15$ & \multirow{2}{*}{0.648} \\
\cline { 2 - 3 } & Control & $30.59 \pm 5.69$ & \\
\cline { 2 - 4 } & Autologous bone + PRGF & $40.63 \pm 14.74$ & \\
\cline { 2 - 4 } & Autologous bone & $46.04 \pm 10.36$ & \\
\hline
\end{tabular}

study, autologous grafting was associated to PRGF in the New Zealand rabbit.

The PRGF used in this study was obtained following the protocol described by Anitua (1999). PRGF was chosen because the activator is calcium chloride, which eliminates the risk of immune reactions and the transmission of diseases associated with the use of exogenous bovine thrombin. Furthermore, PRGF can be obtained in a single centrifugation step at $460 \mathrm{~g}$ for 8 minutes. In contrast, the double centrifugation technique used to obtain PRP requires a greater blood volume (minimum $50 \mathrm{ml}$ ), which is unfeasible in rabbits.

PRGF offers a number of advantages: it allows the simultaneous action of multiple growth factors, and is an autologous product. PRGF also increases tissue vascularization. The product is biocompatible, effective, and safe, and is reabsorbed by the body within days after initiating local regeneration. ${ }^{1-4} \mathrm{de}$ Obarrio et al. ${ }^{15}$ (2000) considered not only the positive effects of PRP resulting from the release of GFs but also its physical and chemical properties.

Gerard et al. ${ }^{11}$ (2006), in an experimental model, found PRP to increase bone formation and remodeling in the first and second months, though these beneficial effects decreased after three and 6 months.

Our results coincide with those reported by Aghaloo et al. ${ }^{10}$ (2002), who found no statistically significant radiographic or histomorphometric differences in bone formation as a result of the addition of PRP. 

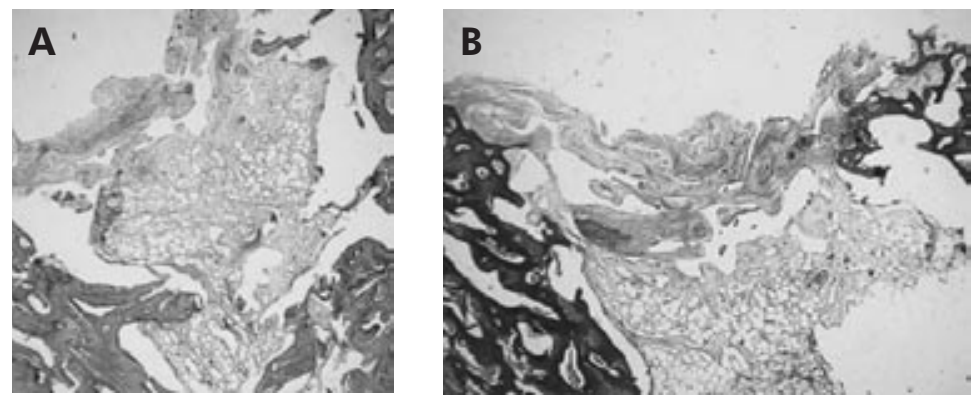

Figure 1 - PRGF treated defect, one month after surgery. (A) Filled defect showing bone marrow at central level (x50, hematoxylin-eosin). (B) Detail of the upper end of the defect, showing neoformed bone and endochondral ossification phenomena (x125, Masson trichromic stain).
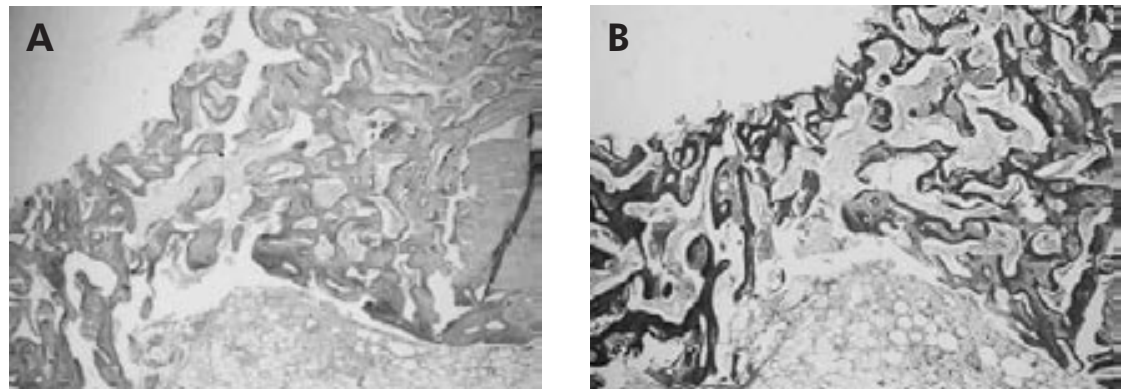

Figure 2 - PRGF treated defect, two months after surgery. (A) Larger-size bone trabeculae (x200, hematoxylineosin). (B) Detail of ossification (x220, Masson trichromic stain).

Our study intervals of one and two months are similar to those published by other authors. ${ }^{14,16}$ Investigators such as Zechner et al. ${ }^{17}$ (2003) suggest a timedependent action on the part of PRP, with a favoring of bone regeneration in the early stages of healing. In our study, certain limitations were imposed by the use of the tibial bone in rabbits instead of the skull. In effect, the defects were limited to $4 \mathrm{~mm}$ in diameter, in order to avoid the risk of fracture.

The data found in the literature regarding bone formation and maturation under the influence of PRP are contradictory. PRP has been combined with autologous bone or bone substitutes such as inorganic bone mineral and organic bone matrix. It remains questionable whether or not these combinations enhance the biological activity of PRP. ${ }^{12}$

According to Pryor et al. ${ }^{14}$ (2006), few studies have used radiographic evaluations in experimental animal models based on triple-point scoring systems such as that used in our study. In effect, most studies make use of dichotomic scores or visual measures based on different tones of gray. ${ }^{18,19}$ In our study, the radiographic analysis showed important variability in the results obtained. In effect, with the scoring system used, significant differences were recorded in radiographic bone density when applying PRGF to the surgical defects, one month after surgery. However, the differences after two months were no longer significant. Pryor et al. ${ }^{14}$ (2006) indicated that while radiography is able to differentiate mineralization patterns, the results should not be overestimated, and histological confirmation is required.

However, in the histomorphometric study, the application of autologous bone either alone or in combination with PRGF did not yield differences in terms of osteogenesis at either of the two time points considered despite a tendency towards increased bone formation compared with the controls.

On the other hand, the group led by Choi et al. ${ }^{20}$ (2004) has suggested that the addition of PRP does not enhance bone formation in autologous bone grafts.

Consensus is lacking as regards the "optimum concentration of the platelet concentrate", despite the existence of a number of studies comparing different PRP production systems. ${ }^{12,13}$ A number of aspects must be taken into account in relation to the preparation of PRP. Although the optimum platelet concentration in PRP has not been established, Weibrich et al. ${ }^{13}$ (2004) suggests that while concentrations below 1,000,000/ $\mu$ l exert suboptimum effects, higher concentrations paradoxically could 
exert inhibitory effects. Other authors consider the concentration of receptors in the target tissue to be more important than the concentration of growth factors in PRP. ${ }^{12}$

\section{Conclusions}

In conclusion, while PRGF either alone or in

\section{References}

1. Anitua E, Sánchez M, Orive G, Andía I. The potential impact of the preparation of plasma rich in growth factors (PRGF) in different medical fields. Biomaterials. 2007;28(31):4551-60.

2. Anitua E, Sánchez M, Nurden A, Nurden P, Orive G, Andía I. New insights into and novel applications for platelet-rich fibrin therapies. Trends in Biotechnol. 2006;24(5):227-34.

3. Marx RE, Carlson ER, Eichstaedt RM, Schimmele SR, Strauss JE, Georgeff KR. Platelet-rich plasma: growth factor enhancement for bone grafts. Oral Surg Oral Med Oral Pathol Oral Radiol Endod. 1998;85(6):638-46.

4. Anitua E. Plasma rich in growth factors: preliminary results of use in the preparation of future sites for implants. Int J Oral Maxillofac Implants. 1999;14(4):529-35.

5. Steed DL. The role of growth factors in wound healing. Surg Clin North Am. 1997;77(3):575-86.

6. Marx RE. Platelet-rich plasma (PRP): what is PRP and what is not PRP? Implant Dent. 2001;10(4):225-8.

7. Zhu SJ, Choi BH, Jung JH, Lee SH, Huh JY, You TM et al. A comparative histologic analysis of tissue-engineered bone using platelet-rich plasma and platelet-enriched fibrin glue. Oral Surg Oral Med Oral Pathol Oral Radiol Endod. 2006;102(2):175-9.

8. Lindeboom JA, Mathura KR, Aartman IHA, Kroon FHM, Milstein DMJ, Ince C. Influence of the application of platelet enriched plasma in oral mucosal wound healing. Clin Oral Impl Res. 2007;18(1):133-9.

9. Wiltfang J, Kloss FR, Kessler P, Nkenke E, Schultre-Mosgau $\mathrm{S}$, Zimmermann $\mathrm{R}$ et al. Effects of platelet-rich plasma on bone healing in combination with autogenous bone and bone substitutes in critical-size defects. An animal experiment. Clin Oral Impl Res. 2004;15(2):187-93.

10. Aghaloo TL, Moy PK, Freymiller EG. Investigation of platelet-rich plasma in rabbit cranial defects: A pilot study. J Oral Maxillofac Surg. 2002;60(10):1176-81.

11. Gerard D, Carlson ER, Gotcher JE, Jacobs M. Effects of platelet-rich plasma on the healing of autologous bone combination with autologous bone appears to have increased new bone formation in our experimental model, statistical significance was not observed. Thus, the application of PRGF in bone defects produced in New Zealand rabbits presented only a limited potential in terms of local bone formation.

grafted mandibular defects in dogs. J Oral Maxillofac Surg. 2006;64(3):443-51.

12. Messora MR, Nagata MJH, Dornelles RC, Bomfim SR, Furlaneto FA, de Melo LG et al. Bone healing in criticalsize defects treated with platelet-rich plasma: a histologic and histometric study in rat calvaria. J Periodontal Res. 2008;43(6):723-9.

13. Weibrich G, Hansen T, Kleis W, Buch R, Hitzler WE. Effect of platelet concentration in platelet-rich plasma on peri-implant bone regeneration. Bone. 2004;34(4):665-71.

14. Pryor ME, Susin C, Wikesjö UME. Validity of radiographic evaluations of bone formation in a rat calvaria osteotomy defect model. J Clin Periodontol. 2006;33(6):455-60.

15. de Obarrio JJ, Araúz-Dutari JI, Chamberlain JM, Croston A. The use of autologous growth factors in periodontal surgical therapy: platelet gel biotechnology - Case reports. Int J Periodontics Restorative Dent. 2000;20(5):486-97.

16. Fuerst G, Gruber R, Tangl S, Sanroman F, Watzek G. Effects of fibrin sealant protein concentrate with and without platelet-released growth factors on bony healing of cortical mandibular defects. An experimental study in minipigs. Clin Oral Implants Res. 2004;15(3):301-7.

17. Zechner W, Tangl S, Tepper G, Fürst G, Bernhart T, Haas R et al. Influence of platelet-rich plasma on osseous healing of dental implants: A histologic and histomorphometric study in minipigs. Int J Oral Maxillofac Implants. 2003;18(1):15-22.

18. Cacciafesta V, Dalstra M, Bosch C, Melsen B, Andreassen TT. Growth hormone treatment promotes guided bone regeneration in rat calvarial defects. Eur J Orthod. 2001;23(6):73340.

19. Hollinger JO, Schmitz JP, Mizgala, JW, Hassler C. An evaluation of two configurations of tricalcium phosphate for treating craniotomies. J Biomed Mater Res. 1989;23(1):17-29.

20. Choi BH, Im CJ, Huh JY, Suh JJ, Lee SH. Effect of plateletrich plasma on bone regeneration in autogenous bone graft. Int J Oral Maxillofac Surg. 2004;33(1):56-9. 